\title{
Radiation-induced Vascular Damage and the Impact on the Treatment Outcome of Stereotactic Body Radiotherapy
}

\author{
EMELY KJELLSSON LINDBLOM ${ }^{1}$, SUSANTA HUI ${ }^{2}$, JAMISON BROOKS ${ }^{2,3}$, \\ ALEXANDRU DASU ${ }^{4,5}$, MACIEJ KUJAWSKI ${ }^{6}$ and IULIANA TOMA-DASU ${ }^{1,7}$ \\ ${ }^{1}$ Medical Radiation Physics, Department of Physics, Stockholm University, Stockholm, Sweden; \\ ${ }^{2}$ Department of Radiation Oncology, Beckman Research Institute of City of Hope, Duarte, CA, U.S.A.; \\ ${ }^{3}$ Department of Radiation Oncology, University of Minnesota, Minneapolis, MI, U.S.A.; \\ ${ }^{4}$ The Skandion Clinic, Uppsala, Sweden; \\ ${ }^{5}$ Department of Immunology, Genetics and Pathology, Uppsala University, Uppsala, Sweden; \\ ${ }^{6}$ Department of Molecular Imaging and Therapy, Beckman Research Institute of City of Hope, Duarte, CA, U.S.A.; \\ ${ }^{7}$ Department of Oncology and Pathology, Karolinska Institutet, Stockholm, Sweden
}

\begin{abstract}
Background/Aim: The aim of this study was to investigate radiation-induced tumour vascular damage and its impact thereof on the outcome of stereotactic body radiotherapy (SBRT). Materials and Methods: Vessel densities in animal tumours before and after a single dose of 20 Gy were quantified and used as input for simulations of three-dimensional tumours with heterogeneous oxygenation. SBRT treatments of the modelled tumours in 1-8 fractions were simulated. The impact of vessel collapse on the outcome of SBRT was investigated by calculating tumour control probability (TCP) and the dose required to obtain a TCP of 50\% $\left(D_{50}\right)$. Results: A radiationinduced increase of acute hypoxia in tumours during SBRT treatment could be simulated based on the experimental data. The $D_{50}$ values for these tumours were higher than for the simulated tumours without vessel collapse. Conclusion: The vascular changes after high doses of radiation could compromise the outcome of SBRT by increasing tumour hypoxia.
\end{abstract}

The modern practice of fractionated radiotherapy is largely based on the so-called 5 R's of radiobiology: repair, repopulation, redistribution, reoxygenation and radiosensitivity (1). For stereotactic treatments such as stereotactic radiosurgery (SRS) and stereotactic body radiotherapy (SBRT) where the treatment is delivered in only one or few fractions, the validity

This article is freely accessible online.

Correspondence to: Emely Kjellsson Lindblom, Medical Radiation Physics, Karolinska University Hospital Solna, S-171 76 Solna, Sweden. Tel: +46 764951628, e-mail: emely.lindblom@fysik.su.se

Key Words: Hypoxia, radiation-induced vascular damage, stereotactic body radiotherapy, SBRT, tumour control probability, TCP, tumour oxygenation. and relevance of the $5 \mathrm{R}$ 's has been questioned $(2,3)$. For example, the steep dose gradients characteristic of stereotactic treatments greatly reduces the impact of normal tissue sparing classically expected from an increased number of fractions (4), while tumour cell repopulation during treatment can be neglected given the short overall treatment time in treatments in few fractions. Furthermore, the effect of redistribution can be disregarded given that high doses are likely to cause cellcycle arrest (5). The limited treatment time and low number of fractions also imply that reoxygenation is limited, indicating that hypoxia has an even bigger impact on the outcome of SRS and SBRT compared to conventionally fractionated radiotherapy $(3,6,7)$. While slow reoxygenation following chronic hypoxia is typically associated with tumour shrinkage, it is highly unlikely to happen during a hypofractionated treatment course where fast reoxygenation of acute hypoxia is expected to occur between individual fractions (8). Reoxygenation, and the closely related radiosensitivity of tumour cells, thus remain highly influential parameters in stereotactic radiotherapy. Recently, there has been an increasing interest in the study of additional effects occurring only at high doses per fraction, particularly the damage to the tumour vasculature. Based on experimental observations, it has been suggested that damage to the tumour vasculature could have a secondary effect resulting from the sudden loss of blood supply and/or from an increase in the radiosensitivity of tumour cells due to a cytotoxic effect derived from endothelial apoptosis (2, $9,10)$. While the vascular effect has, thus, almost exclusively been discussed as a potential second cytotoxic factor for tumour cells, the observed vascular response after doses of 1015 Gy appears to be highly dynamic (2). With an initial reduction in blood flow that persists for various lengths of time, the actual vascular effect with respect to the treatment efficacy could therefore be highly dependent on the time point at which treatment fractions are delivered with respect to each other. 
Therefore, instead of increased cell death, increased radioresistance could be expected during the limited time of e.g. an SBRT treatment if the percentage of acutely hypoxic cells is temporarily increased from one fraction to the next. Thus, there is a gap in understanding how dose-fractionation actually affects the tumor vascular microenvironment, and impacts on the tumor control. In this study, radiobiological experiments and modelling were combined to investigate the changes in the tumour vasculature resulting from high doses and the subsequent impact on the tumour control probability (TCP) in stereotactic radiotherapy treatments.

\section{Materials and Methods}

The study consisted of two parts, one experimental part in which data on tumour vasculature before and after a dose of 20 Gy was collected and analysed, and one modelling part in which the experimental data was used to simulate the effect from the observed vascular response at the level of the whole tumor.

Mouse irradiation, multiphoton imaging and analysis. All procedures for animal experiments were performed according to City of Hope guidelines and approved by the Institutional Animal Care and Use Committee. Four NOD/SCID 16-18 week old female mice were injected with 105 E0771 CEA+ breast cancer cells suspended $50 \mu \mathrm{l}$ PBS and matrigel in equal parts. Cells were injected onto the mammary fat pad. Two of the mice were anaesthetised and treated with $20 \mathrm{~Gy}$ using a targeted image-guided dose-calculated irradiation system (Precision X-Ray, North Branford, CT, USA) (11). Treatment was performed 10 days post E0771 injection. One day post treatment, mice were anesthetized initially with $3 \%$ isoflurane at a flow rate of $21 / \mathrm{min}$ mice. Retro-orbital injections of $500 \mu \mathrm{g}$ of $70 \mathrm{kDa}$, FITC-conjugated fluorescent Dextran (Sigma Aldrich, St. Louis, MO, USA) suspended in $100 \mu \mathrm{l}$ PBS were given to mice for imaging tumourvascular lumen. Mice were placed on a custom built heated stage maintained at $37^{\circ} \mathrm{C}$, and anaesthesia was reduced to $1.5 \%$ isoflurane at a flow rate of $1.5 \mathrm{l} / \mathrm{min}$ for surgery and imaging. A midline incision to the skin was made. The skin, tumour, and mammary fat pad were separated from the peritoneum preserving vasculature surrounding the tumour. A glass coverslip was placed over the upturned mammary fat pad and tumour to enable proper imaging. Phosphate buffered saline was applied under the coverslip to preserve tissue function. Multiphoton microscopy was performed using a Prairie Ultima multiphoton microscope (Bruker Corporation Billica, MA, USA). Fluorescent excitation was performed using a Chameleon Ultra II tunable Ti:Sapphire laser with 140 femtosecond pulses (Coherent, Santa Clara, CA, USA). A 10x objective (UPlanFL N 10X/0.30 $\infty /-/ F N 26.5$, Olympus, Tokyo, Japan) was used to acquire all images. Tiled three-dimensional images were acquired by collecting a grid series of $15 \%$ overlapping z-stack images with a slice spacing of $15 \mu \mathrm{m}$. Data acquisition using the Prairie Ultima microscope was handled by Prairieview 5.3 software. Tiled images were stitched together using a Fiji/ImageJ grid collection/stitching plugin $(12,13)$. Fiji/ImageJ was used to manually quantify vessels in the tumour. Vessel branches were counted from three dimensional images manually and normalised for image area. Six square subregions of approximately $0.85 \mathrm{~mm}^{2}$ were analysed for each tumour. The regions were then used for measurements of intra-tumour vessel density variation including the calculation of the median, mean and standard deviation in each tumour.
Radiobiological modelling. To simulate the effect of radiation given in few fractions from the experimentally observed vascular responses at the level of the whole tumor, a previously developed three-dimensional in silico tumour model of heterogeneous tumour oxygenation and radiation response was used (14). The input to this model is distributions of inter-vessel distances (IVDs) previously obtained through corrosion casting in animal solid tumours (15). In the model, the tumour is geometrically defined including potential sub-volumes to which different IVDs can be assigned. In this way, several IVDs distributions (with mean values of 60-160 $\mu \mathrm{m}$ ) can be combined in the same tumour to simulate tumours with a broad range of oxygenation distributions. Blood vessel capillaries are randomly placed in the defined volumes according to the assigned IVDs distribution, and a fraction of the vessels is randomly removed in order to simulate acute hypoxia resulting from a temporary collapse or occlusion of capillaries. The three-dimensional tumour map of oxygen partial pressure $\left(p \mathrm{O}_{2}\right)$ is then calculated by solving a differential equation describing the diffusion and consumption of oxygen from the vessels. Due to the random nature of the exact spatial distribution of active vessels, fast reoxygenation can be simulated by repeating the assignment of IVDs distributions and recalculating the $\mathrm{pO}_{2}$ map (14).

In order to construct in silico tumours representative of the experimental tumours in the present study, different combinations of blood vessel distributions characterised by different IVDs resulting in the experimentally determined mean vessel densities and standard deviations were sought. While the simulations produce three-dimensional models of the whole tumour, the experimental measurements were obtained from a limited number of samples. Therefore, combinations of several IVDs were investigated for the purpose of finding a mean vessel density and standard deviation matching the experimental vessel density assuming equal weights between the IVDs.

In order to investigate the impact of radiation-induced vessel collapse on the treatment outcome, SBRT treatments delivered in 18 fractions were simulated by delivering a homogeneous dose to the modelled tumours representing both the untreated (i.e. without vessel collapse) and treated (with vessel collapse) experimental tumours. In order to construct dose-response curves, a range of doses was considered. The cell surviving fraction $S F_{i}$ in each voxel $i$ was subsequently calculated using the linear-quadratic (LQ) model taking into account the $p \mathrm{O}_{2}$ on voxel level by modifying the radiosensitivity parameters $\alpha$ and $\beta$ of the LQ model with oxygen modifying factors (OMFs) $(14,16)$ :

$$
S F_{i}=\exp \left(-\frac{\alpha}{O M F_{i}} \cdot d_{i}-\frac{\beta}{O M F_{i}^{2}} \cdot d_{i}^{2}\right)
$$

In order to evaluate the simulated treatments, the tumour control probability (TCP) was calculated as the product of the individual TCPs in each voxel, assuming a homogeneous density $N$ of clonogenic cells at the start of the treatment:

$$
T C P=\prod_{i=1}^{N v o x} \exp \left(-N \cdot S F_{i}\right)
$$


The parameter values used in the simulations were $\alpha=0.35 \mathrm{~Gy}^{-1}$, $\beta=0.035 \mathrm{~Gy}^{-2}(\alpha / \beta=10 \mathrm{~Gy}), O M F_{\max }=3$ and $N=10^{8}$ cells $(17)$.

\section{Results}

Figure 1 and Table I show the experimentally determined vessel densities in four animal tumours. Both the highest and lowest average vessel density in the two untreated tumours were found to be $18.0 \mathrm{~mm}^{-2}$ and $138.0 \mathrm{~mm}^{-2}$, respectively. The average vessel densities in the treated tumours were between $71.7 \mathrm{~mm}^{-2}$ and $62.0 \mathrm{~mm}^{-2}$. In the untreated tumour with low average vessel density, the six samples were extremely similar in comparison with the considerable heterogeneity observed in the three other tumours (Figure 1). Given the well-known heterogeneous nature of tumour vasculature, this could indicate that the six samples are less representative of the tumour vasculature in the case of tumour 1 , and the simulated tumour before treatment was therefore based on the data for tumour 2. Based on the comparison of the IVDs with the experimental results for the two treated tumours, the simulation of tumours after a high dose was based on the data for tumour 4 . Two patterns of hypoxia representative of clinical findings were considered: one in which the tumour oxygenation decreases towards the centre of the tumour, and one with a more heterogeneous pattern of multiple islands not located in the centre of the tumour (18). Figure 2 shows the cross sections through the simulated tumours with and without radiation-induced vessel collapse.

Figure 2A and B should thus be considered to represent the same tumour with and without radiation-induced vessel collapse according to the experimental findings (and the same for the tumours illustrated in Figure 2C and D).

Figure 3 shows the dose-response curves obtained for the tumours with central hypoxia, and Figure 4 shows the doseresponse curves obtained for the tumours in which islands ofhypoxia were present.

In Table II, the $\mathrm{D}_{50}$ values of the dose-response curves in Figures 3 and 4 are presented.

The values of $D_{50}$ are in general agreement with the doses clinically prescribed in SBRT $(19,20)$. Furthermore, the $\mathrm{D}_{50}$ is higher for the tumours with radiation-induced vessel collapse as a result of the fact that they are more hypoxic.

\section{Discussion}

With an increasing use of stereotactic body radiotherapy and other advanced radiotherapy techniques employing fundamentally different dose distributions and fractionation schedules, the classical 5 R's describing the response of lateand early-reacting tissues to fractionated radiotherapy have been challenged in recent years. Several experimental and clinical studies have reported interesting observations of

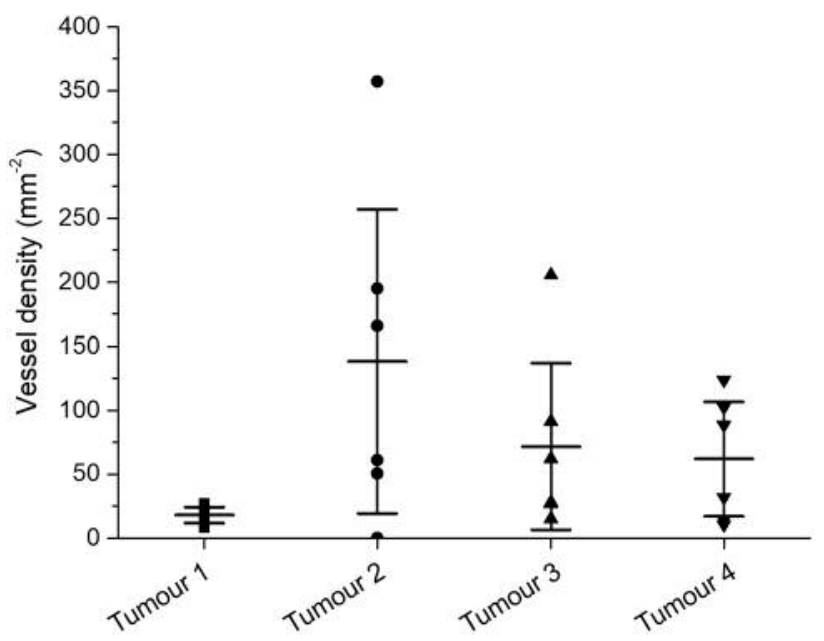

Figure 1. Vessel densities in the six subregions for each of the experimental tumours.

Table I. The mean value of vessel density in each tumour with the corresponding standard deviation (Stdv) and coefficient of variance $(\mathrm{CV})$.

\begin{tabular}{lrrrr}
\hline & \multicolumn{2}{c}{ No treatment } & \multicolumn{2}{c}{$20 \mathrm{~Gy}$} \\
\cline { 2 - 5 } Tumour & \multicolumn{1}{c}{1} & 2 & \multicolumn{1}{c}{3} & \multicolumn{1}{c}{4} \\
\hline Mean $\left[\mathrm{mm}^{-2}\right]$ & 18.0 & 138.0 & 71.7 & 62.0 \\
Stdv $\left[\mathrm{mm}^{-2}\right]$ & 7.0 & 130.0 & 71.5 & 49.1 \\
CV (stdv/mean) $\left[\mathrm{mm}^{-2}\right]$ & 0.4 & 0.9 & 1.0 & 0.8 \\
\hline
\end{tabular}

Stdv: Standard deviation; CV: coefficient of variance.

effects that seem to occur only at high doses per fraction. In melanoma patients treated with a combination of high-dose radiotherapy and the monoclonal antibody ipilimumab, an abscopal effect was observed as lesions outside of the radiation field regressed after the treatment (21). This has been suggested to be a result of enhanced anti-tumour immunity after irradiation, but the rather limited clinical findings so far warrant further investigation of whether this phenomenon is specifically attributed to SBRT-like treatments and if other tumour types besides melanoma exhibit the effect (3). In addition to an interesting potential interplay with the immune system, the administration of high doses has been associated with damage to the tumour vasculature which might have a secondary effect in stereotactic treatments. This has been hypothesised to happen for two reasons: secondary tumour cell death from the sudden loss of blood supply and increased radiosensitivity of tumour cells due to a cytotoxic effect derived from endothelial apoptosis $(2,9,10)$. While the discussion has 
A Tumour 2

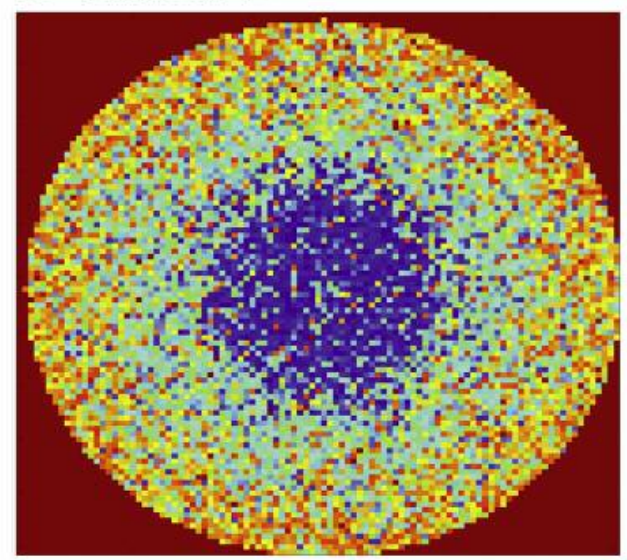

C Tumour 2

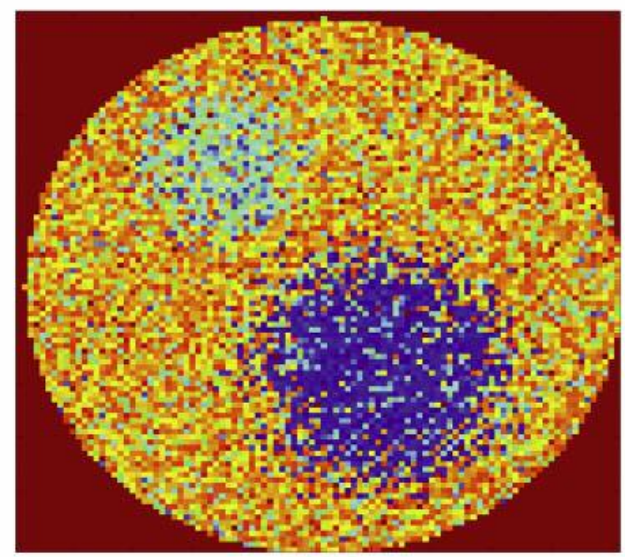

B Tumour 4

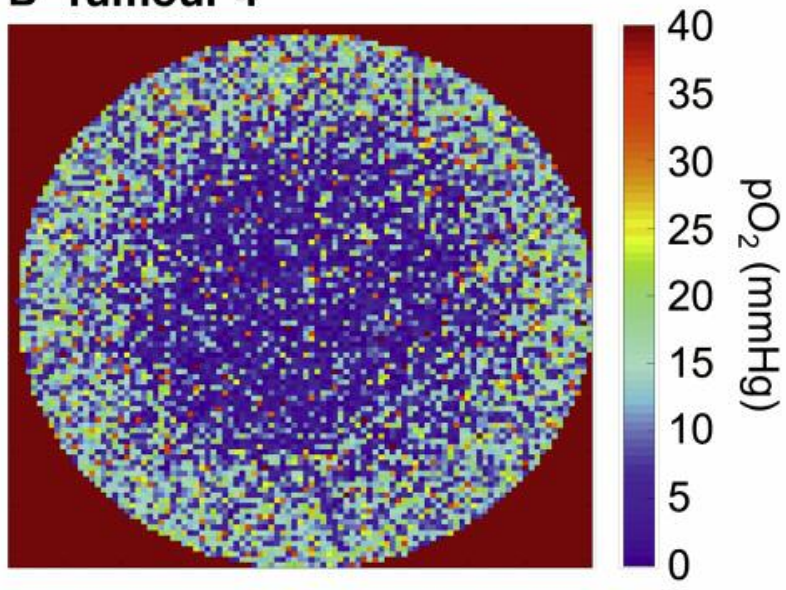

\section{Tumour 4}

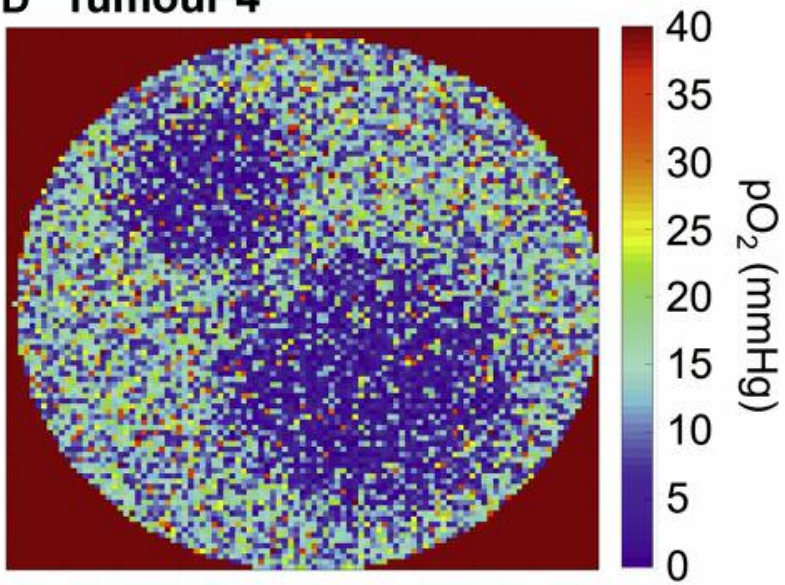

Figure 2. Illustration of (A) simulated tumour without vessel collapse, hypoxic fraction $\mathrm{HF}<5 \mathrm{mmHg}=5 \%$, (B) corresponding tumour with vessel collapse, $H F=25 \%,(C)$ simulated tumour without vessel collapse, hypoxic fraction $H F=5 \%,(D)$ corresponding tumour with vessel collapse, $H F=15 \%$.

been focused on a contribution to cell death, it should be pointed out that vessel collapse also occurs spontaneously in tumours, causing perfusion-limited acute hypoxia (8). This leads to an increased resistance to radiation, as opposed to cell death from acute deprivation of nutrients. Acute hypoxia is a transient state that can be reversed by fast reoxygenation before the affected cells are permanently damaged. If radiation-induced vessel collapse can also be reversed in due time, an increase in the radioresistance of the tumour could thus equally well be expected following a high dose. In fact, radiation-induced damage to the tumour vasculature has been observed to be repaired and the vasculature is restored (2). This indicates that the actual vascular effect could imply a reduction rather than an increase in tumour cell death, and highlights the importance of the timing of e.g. SBRT fractions. A recent modelling study demonstrated this effect for an assumed vessel collapse of $35 \%$ caused by the first fraction and persisting through the remaining treatment (22). This led to a greater loss of tumour control probability than of double sized chronically hypoxic tumour compartment.

In the present study, the response of the tumour vasculature to a high dose was studied experimentally, and the therapeutic implications thereof were investigated by radiobiological modelling based on the experimental data. Four model tumours representative of the experimental tumours were simulated, two rather well-oxygenated tumours with no presumed vessel collapse, and two more hypoxic tumours with radiation-induced vessel collapse. The $\mathrm{D}_{50}$ values of all simulated tumours were in agreement with the clinically prescribed doses in SBRT, with the exception of the single-fraction schedules in line with previous findings (6). For the tumours with vessel collapse, the $\mathrm{D}_{50}$ was consistently higher than that in the simulated tumours without vessel collapse.The biggest differences were 
A
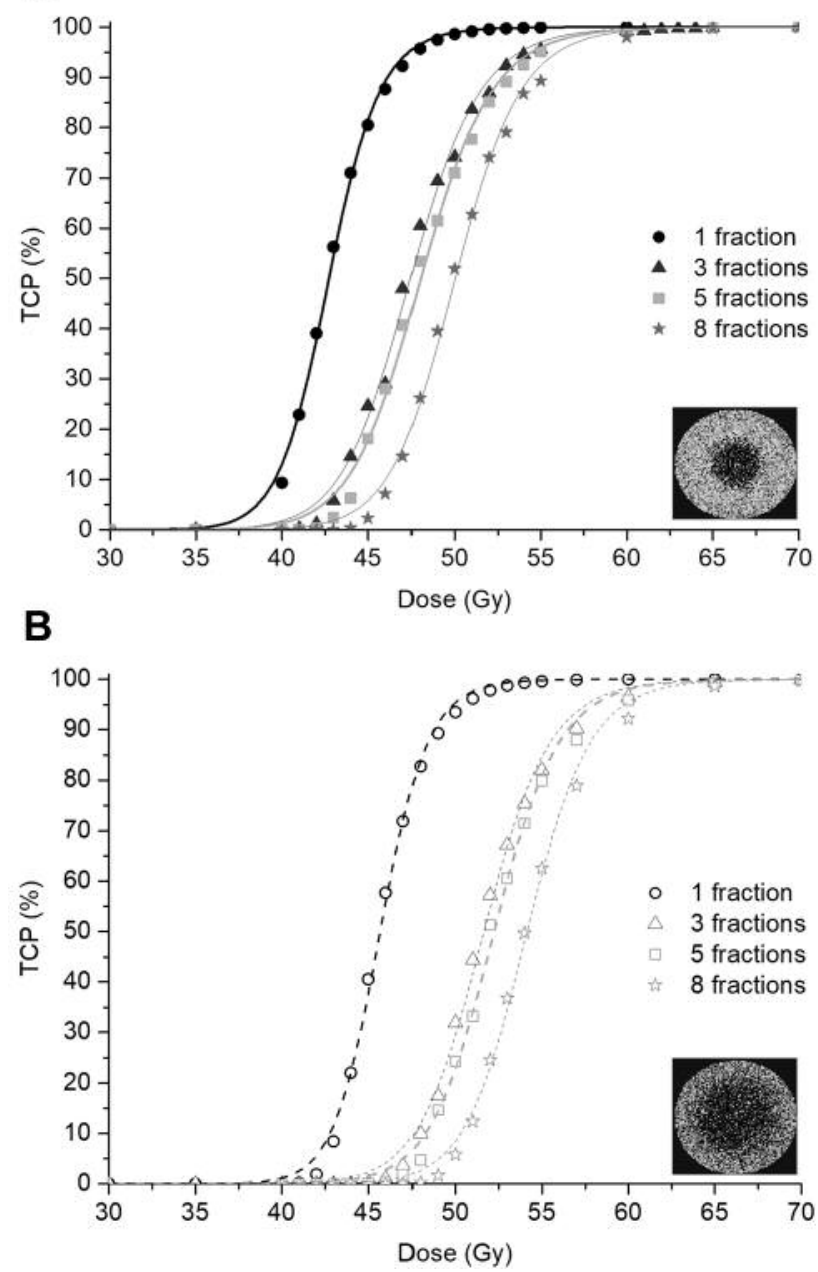

Figure 3. Dose-response curves for the simulated SBRT treatments of 1-8 fractions delivered to a tumour with centralised hypoxia with and without vessel collapse, $(A)$ and $(B)$ respectively.
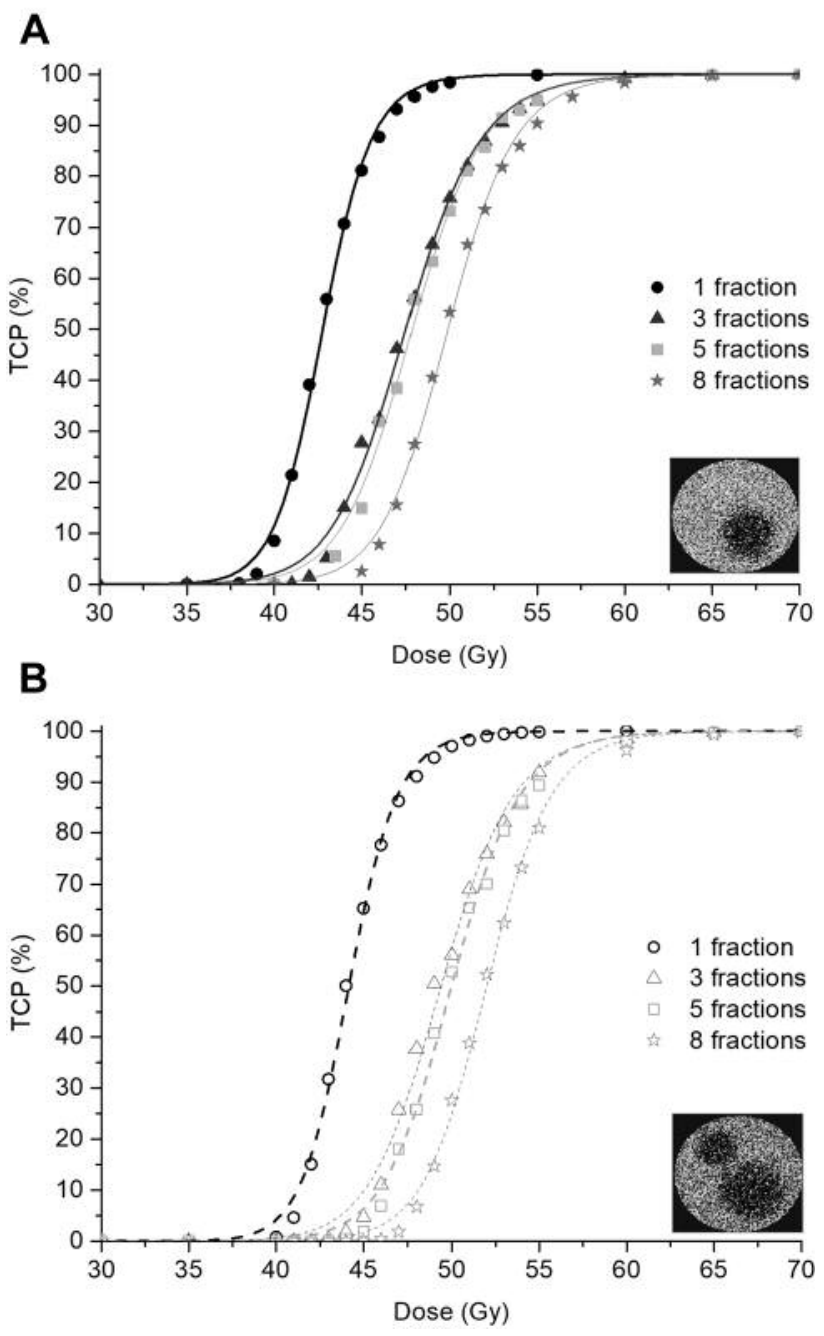

Figure 4. Dose-response curves for the simulated SBRT treatments of 1-8 fractions delivered to a tumour with multi-island hypoxia with and without vessel collapse, $(A)$ and $(B)$ respectively.

Table II. Tumour control probability (TCP) for the simulated treatments.

\begin{tabular}{|c|c|c|c|c|}
\hline \multirow[t]{2}{*}{ Central hypoxia } & \multicolumn{4}{|c|}{$\mathrm{D}_{50}(\mathrm{~Gy})$} \\
\hline & 1 Fraction & 3 Fractions & 5 Fractions & 8 Fractions \\
\hline No radiation-induced vessel collapse, $\mathrm{HF}=5 \%$ & 42.7 & 47.4 & 48.0 & 50.0 \\
\hline Radiation-induced vessel collapse, $\mathrm{HF}=25 \%$ & 45.7 & 51.6 & 52.2 & 54.1 \\
\hline \multirow[t]{2}{*}{ Multi-island hypoxia } & \multicolumn{4}{|c|}{$\mathrm{D}_{50}(\mathrm{~Gy})$} \\
\hline & 1 Fraction & 3 Fractions & 5 Fractions & 8 Fractions \\
\hline No radiation-induced vessel collapse, $\mathrm{HF}=5 \%$ & 42.8 & 47.5 & 47.9 & 49.9 \\
\hline Radiation-induced vessel collapse, $\mathrm{HF}=15 \%$ & 44.2 & 49.4 & 50.0 & 52.0 \\
\hline
\end{tabular}

TCP: Tumour control probability; $\mathrm{D}_{50}$ : dose required to achieve 50\% tumour control probability; HF: hypoxic fraction. 
observed for the most hypoxic tumour with a hypoxic fraction $\leq 5 \mathrm{mmHg}$ of $25 \%$. Although it is well-known that higher dose is required in the more hypoxic tumour (23), the fact that the simulated tumours in this study were based on experimental tumours with and without radiation-induced vessel collapse provides support to the the conclusion that the vascular effect from high doses could lead to hypoxia. The simulations in turn show that this could have an impact on the treatment efficacy of $e . g$. SBRT through an increase in radiation resistance of the tumour.

In the analysis of the experimental data, one of the untreated tumours was excluded due to the very low vessel density and limited spread of sample values. As mentioned in the Results section, this could indicate that the samples acquired were not representative of the vasculature of the tumour, in which a higher degree of heterogeneity would be expected as has been observed in the other experimental tumours and in numerous previous studies $(24,25)$. More importantly, the inclusion of a homogeneously hypoxic tumour as representative of a case without radiation-induced vessel collapse would compromise the purposes of the present study, focusing on the potential detrimental effects resulting from increased tumour hypoxia as a result of significant treatment-induced vascular damage. Furthermore, in the clinical setting, this effect would not be of major (if any) concern for such highly hypoxic tumours, which would be considered high-risk and in potential need for hypoxiatargeted treatment adaptation before the start of the treatment. Nonetheless, the number of tumours included in the experimental part of this study is small, and the measurements of the tumor vasculature were perfomed only $48 \mathrm{~h}$ post-SBRT. In order to expand the analysis and better understand the effects derived from vascular damage following high doses radiation, further experiments and modelling studies are planned in the near future.

In this study, experimental data on the changes in the tumour vasculature following a high dose of radiation were used to simulate tumours with increased acute hypoxia resulting from radiation-induced vessel collapse. Depending on the extent of vessel collapse and the prescribed dose, vascular changes after high doses of radiation could compromise the outcome of SBRT by increasing tumour hypoxia.

\section{Conflicts of Interest}

The Authors have no conflicts of interests to declare.

\section{Authors' Contributions}

EKL performed the simulations and wrote the manuscript. JB and $\mathrm{SH}$ performed the experimental work and subsequent analysis. AD and ITD provided the model for tumour oxygenation and ITD produced input data for the simulations. All Authors reviewed the manuscript.

\section{Acknowledgements}

Research reported in this publication is supported by the National Cancer Institute of the National Institutes of Health under award number P30CA033572 and partly supported by National Institutes of Health grant 1R01CA154491-01. One of the author acknowledges discussion and suggestions provided by Professor John Shively, City of Hope. Financial support from the Cancer Research Funds of Radiumhemmet (Solna, Sweden) is gratefully acknowledged.

\section{References}

1 Mayles P, Nahum A and Rosenwald JC (eds.).: Handbook of Radiotherapy Physics: Theory and Practice. Boca Raton, CRC Press, Taylor \& Francis Group, 2007.

2 Park HJ, Griffin RJ, Hui S, Levitt SH and Song CW: Radiationinduced vascular damage in tumors: implications of vascular damage in ablative hypofractionated radiotherapy (SBRT and SRS). Radiat Res 177(3): 311-327, 2012. PMID: 22229487.

3 Brown JM, Carlson DJ and Brenner DJ: The tumor radiobiology of SRS and SBRT: are more than the 5 Rs involved? Int J Radiat Oncol Biol Phys 88(2): 254-262, 2014. PMID: 24411596. DOI: 10.1016/j.ijrobp.2013.07.022

4 Yang J, Fowler JF, Lamond JP, Lanciano R, Feng J and Brady LW: Red shell: defining a high-risk zone of normal tissue damage in stereotactic body radiation therapy. Int J Radiat Oncol Biol Phys 77(3): 903-909, 2010. PMID: 20400240. DOI: 10.1016/j.ijrobp.2009.12.069

5 Song CW, Park H, Griffin RJ and Levitt SH: Radiobiology of stereotactic radiosurgery and stereotactic body radiation therapy. In: Technical Basis of Radiation Therapy. Berlin, Heidelberg, Springer, pp. 51-61, 2011.

6 Lindblom E, Antonovic L, Dasu A, Lax I, Wersäll P and TomaDasu I: Treatment fractionation for stereotactic radiotherapy of lung tumours: a modelling study of the influence of chronic and acute hypoxia on tumour control probability. Radiat Oncol 9: 149, 2014. PMID: 24974778. DOI: 10.1186/1748-717X-9-149

7 Toma-Dasu I, Sandström H, Barsoum P and Dasu A: To fractionate or not to fractionate? That is the question for the radiosurgery of hypoxic tumors. J Neurosurg 121: 110-115, 2014. PMID: 25434944. DOI: 10.3171/2014.8.GKS141461

8 Ljungkvist AS, Bussink J, Kaanders JH and van der Kogel AJ: Dynamics of tumor hypoxia measured with bioreductive hypoxic cell markers. Radiat Res 167(2): 127-145, 2007. PMID: 17390721.

9 Garcia-Barros M, Paris F, Cordon-Cardo C, Lyden D, Rafii S, Haimovitz-Friedman A, Fuks Z and Kolesnick R: Tumor response to radiotherapy regulated by endothelial cell apoptosis. Science 300(5622): 1155-1159, 2003. PMID: 12750523. DOI: 10.1126/science. 1082504

10 Fuks Z and Kolesnick R: Engaging the vascular component of the tumor response. Cancer Cell 8(2): 89-91, 2005. PMID: 16098459. DOI: $10.1016 /$ j.ccr.2005.07.014

11 Van Hoof SJ, Granton PV and Verhaegen F: Development and validation of a treatment planning system for small animal radiotherapy: SmART-Plan. Radiother Oncol 109(3): 361-366, 2013. PMID: 24183860. DOI: 10.1016/j.radonc.2013.10.003

12 Schindelin J, Arganda-Carreras I, Frise E, Kaynig V, Longair M, Pietzsch T, Preibisch S, Rueden C, Saalfeld S, Schmid B, 
Tinevez JY, White DJ, Hartenstein V, Eliceiri K, Tomancak P and Cardona A: Fiji: an open-source platform for biologicalimage analysis. Nat Methods 9(7): 676-682, 2012. PMID: 22743772. DOI: $10.1038 /$ nmeth 2019

13 Preibisch S, Saalfeld S and Tomancak P: Globally optimal stitching of tiled 3D microscopic image acquisitions. Bioinformatics 25(11): 1463-1465, 2009. PMID: 19346324. DOI: $10.1093 /$ bioinformatics/btp184

14 Toma-Dasu I and Dasu A: Modelling tumour oxygenation, reoxygenation and implications on treatment outcome. Comput Math Methods Med 2013: 141087, 2013. PMID: 23401721. DOI: $10.1155 / 2013 / 141087$

15 Konerding MA, Malkusch W, Klapthor B, van Ackern C, Fait E, Hill SA, Parkins C, Chaplin DJ, Presta M and Denekamp J: Evidence for characteristic vascular patterns in solid tumours: quantitative studies using corrosion casts. Br J Cancer 80(5-6): 724-732, 1999. PMID: 10360650. DOI: 10.1038/sj.bjc.6690416

16 Alper T and Howard-Flanders P: Role of oxygen in modifying the radiosensitivity of E. coli B. Nature 178(4540): 978-979, 1956. PMID: 13378491.

17.Lindblom E, Dasu A and Toma-Dasu I: Optimal fractionation in radiotherapy for non-small cell lung cancer--a modelling approach. Acta Oncol 54(9): 1592-1598, 2015. PMID: 26217986. DOI: $10.3109 / 0284186$ X.2015.1061207

18 Bittner MI and Grosu AL: Hypoxia in head and neck tumors: characteristics and development during therapy. Front Oncol 3: 223, 2013. PMID: 24010122. DOI: 10.3389/fonc.2013.00223

19 Baumann P, Nyman J, Hoyer M, Wennberg B, Gagliardi G, Lax I, Drugge N, Ekberg L, Friesland S, Johansson KA, Lund JA, Morhed E, Nilsson K, Levin N, Paludan M, Sederholm C, Traberg A, Wittgren L and Lewensohn R: Outcome in a prospective phase II trial of medically inoperable stage I nonsmall-cell lung cancer patients treated with stereotactic body radiotherapy. J Clin Oncol 27(20): 3290-3296, 2009. PMID: 19414667. DOI: $10.1200 / J C O .2008 .21 .5681$

20 Takeda A, Sanuki N, Kunieda E, Ohashi T, Oku Y, Takeda T, Shigematsu $\mathrm{N}$ and Kubo A: Stereotactic body radiotherapy for primary lung cancer at a dose of $50 \mathrm{~Gy}$ total in five fractions to the periphery of the planning target volume calculated using a superposition algorithm. Int J Radiat Oncol Biol Phys 73(2): 442-448, 2009. PMID: 18990507. DOI: 10.1016/j.ijrobp. 2008.04 .043
21 Postow MA, Callahan MK, Barker CA, Yamada Y, Yuan J, Kitano S, Mu Z, Rasalan T, Adamow M, Ritter E, Sedrak C, Jungbluth AA, Chua R, Yang AS, Roman RA, Rosner S, Benson B, Allison JP, Lesokhin AM, Gnjatic S and Wolchok JD: Immunologic correlates of the abscopal effect in a patient with melanoma. N Engl J Med 366(10): 925-931, 2012. PMID: 22397654. DOI: 10.1056/NEJMoa1112824

22 Kjellsson Lindblom E, Dasu A and Toma-Dasu I: Hypoxia induced by vascular damage at high doses could compromise the outcome of radiotherapy. Anticancer Res 36(5): 2337-2340, 2019. DOI: 10.21873 /anticanres. 13350

23. Epel B, Maggio MC, Barth ED, Miller RC, Pelizzari CA, Krzykawska-Serda M, Sundramoorthy SV, Aydogan B, Weichselbaum RR, Tormyshev VM and Halpern HJ: Oxygenguided radiation therapy. Int J Radiat Oncol Biol Phys 103(4): 977-984, 2019. PMID: 30414912. DOI: 10.1016/j.ijrobp.2018. 10.041

24 Koh WJ, Bergman KS, Rasey JS, Peterson LM, Evans ML, Graham MM, Grierson JR, Lindsley KL, Lewellen TK, Krohn, KA and Griffin TW: Evaluation of oxygenation status during fractionated radiotherapy in human nonsmall cell lung cancers using [F-18]fluoromisonidazole positron emission tomography. Int J Radiat Oncol Biol Phys 33(2): 391-398, 1995. PMID: 7673026. DOI: 10.1016/0360-3016(95)00170-4

25 Rasey JS, Koh WJ, Evans ML, Peterson LM, Lewellen TK, Graham MM and Krohn KA: Quantifying regional hypoxia in human tumors with positron emission tomography of [18F]fluoromisonidazole: a pretherapy study of 37 patients. Int J Radiat Oncol Biol Phys 36(2): 417-428, 1996. PMID: 8892467.
Received May 4, 2019

Revised May 15, 2019

Accepted May 16, 2019 